\title{
On the application of simple rift basin models to the south polar region of Enceladus
}

\author{
C. C. Walker, ${ }^{1}$ J. N. Bassis, ${ }^{1}$ and M. W. Liemohn ${ }^{1}$ \\ Received 16 March 2012; revised 24 May 2012; accepted 2 June 2012; published 14 July 2012.
}

[1] The south polar terrain (SPT) of Saturn's moon Enceladus is a mysteriously active region that exhibits intriguing tectonic signatures and widespread fracturing. The central region of the nearly-circular SPT is depressed into the surface by a few hundred meters and bounded by a ring of cliffs roughly $1 \mathrm{~km}$ high. In this study, we investigate whether this depression and surrounding mountainous uplift is consistent with the morphology of terrestrial rift basins and the possibility that the SPT could have formed during a tectonic event analogous to those of such rift basins on Earth. Using three mechanical models of basin formation, we compare our predicted topography of the SPT with observed topography of the region. The first of three models we consider assumes crustal stretching by factor $\beta$, and predicts a basin depth of roughly $600 \mathrm{~m}$, closely matching previously published estimates of the depth at the SPT. Models of extension and compression, assuming an elastic response in the ice crust, predict best-fit mountain uplift of roughly $1820 \mathrm{~m}$ and $1130 \mathrm{~m}$, respectively. Our preferred model suggests that the icy shell in the SPT has been stretched, but the extension is (partially) balanced by compression along the edges of the basin leading to the uplift of the mountains along the boundary, thereby implying that the SPT may have a tectonic origin analogous to that of a terrestrial basin.

Citation: Walker, C. C., J. N. Bassis, and M. W. Liemohn (2012), On the application of simple rift basin models to the south polar region of Enceladus, J. Geophys. Res., 117, E07003, doi:10.1029/2012JE004084.

\section{Introduction}

[2] Saturn's moon Enceladus is unusual in that it is among only a handful of solar system objects known to be geologically active; a striking characteristic given that the global surface temperature is $70 \mathrm{~K}$ and the moon, covered in an icy shell, has an effective radius of only $250 \mathrm{~km}$ [Porco et al., 2006]. Observations of the icy surface indicate a long history of activity and deformation. Different sections of the surface range in age from primordial (cratered plains regions have estimated age up to 4.2 billion years or 1.7 billion years, depending on impactor flux model used [Spencer et al., 2009]) to geologically recent [Porco et al., 2006; Bland et al., 2007; Barr, 2008]. The variation in surface features and ages across the moon imply that regional, rather than global, resurfacing processes are dominant [Kargel and Pozio, 1996].

[3] The region of interest in this paper is the South Pole Terrain (SPT), the youngest region of the satellite's surface with an age $<10$ Myr [Barr and Preuss, 2010]. The SPT most notably features the "tiger stripe" fractures at its center, along with their associated active plume vents [e.g., Spencer

\footnotetext{
${ }^{1}$ Department of Atmospheric Oceanic and Space Sciences, University of Michigan, Ann Arbor, Michigan, USA.

Corresponding author: C. C. Walker, Department of Atmospheric Oceanic and Space Sciences, University of Michigan, Space Research Building, 2455 Hayward St., Ann Arbor, MI 48105, USA. (catcolwa@umich.edu)

(C)2012. American Geophysical Union. All Rights Reserved. 0148-0227/12/2012JE004084
}

et al., 2009]. Evidence of continual tectonic activity in the region and the observation of the active venting at the South Pole suggest the possibility of a subsurface ocean [e.g., Collins and Goodman, 2007]. The SPT on the whole has been described in detail by Gioia et al. [2007], Spencer et al. [2009], and others; the terrain can be generally classified into the central tiger stripe terrain, which coincides with the underlying thermal anomaly at the South Pole, a slim circular terrain which surrounds the central terrain but has few fractures of its own, and a prominent, concentric ring of ridges. The floor of the region is relatively flat and shallowly depressed relative to the geoid [Thomas et al., 2007; Schenk and McKinnon, 2009]. Estimates of the depth of the floor range from $200 \mathrm{~m}$ [Thomas et al., 2007; Roberts and Nimmo, 2008] to about $500 \mathrm{~m}$ at the center [Helfenstein et al., 2011] and up to about $800 \mathrm{~m}$ [Schenk and McKinnon, 2009], depending on assumed shape models and density structures within the ice, respectively. The area is enclosed by a curved, pole-ward facing cliff system, creating a nearly-circular border to the region at about $55^{\circ} \mathrm{S}$ [Spencer et al., 2009] and has been interpreted as a convergent feature resulting from compressive stress along that boundary in the North-South direction [Porco et al., 2006]. The steep footwalls and gradually sloping flanks have been estimated to be roughly 1 $\mathrm{km}$ in height [e.g., Schenk and McKinnon, 2009; Spencer et al., 2009]. Another significant feature of the SPT manifests itself in a radial set of large rifted features, extending in a "starfish" shape toward the equator [Gioia et al., 2007] (Figure 1). Speculation that the topographic appearance and 


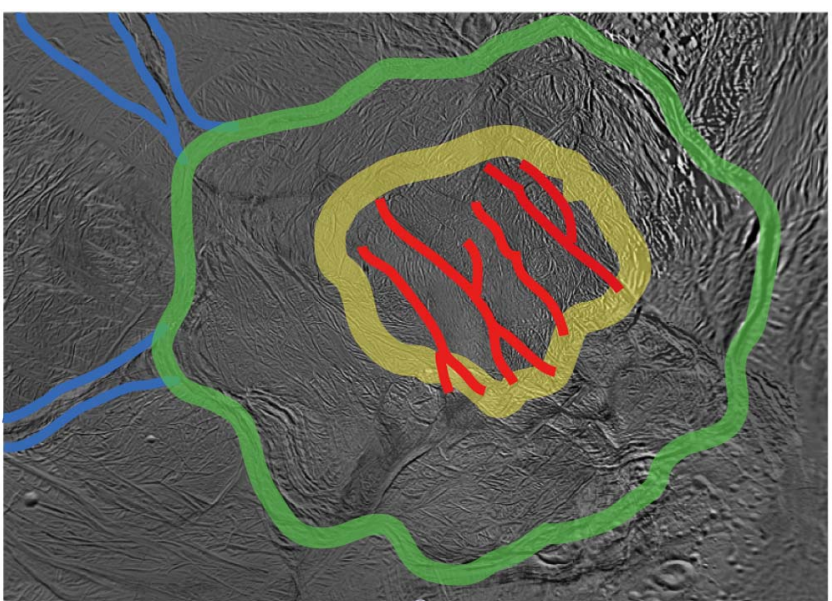

Figure 1. The south polar terrain of Enceladus as imaged by Cassini (NASA/JPL/Space Science Institute). The major sections of topography at the SPT are denoted here: the four main tiger stripes marked in red, within the central depressed tiger stripe terrain outlined in yellow; the surrounding region that appears mottled but has few fractures itself, also depressed, outlined in green; the green outline, thereby, also highlights the location of the mountainous uplift at the boundary of the region. The highest point of uplift is estimated at $1 \mathrm{~km}$. Blue lines radially outward from the SPT mark two of the "starfish arms" - large-scale fracture features radiating away from the SPT toward the equator.

activity at the SPT are manifestations of subsurface dynamics and/or variability in structure is bolstered by the observation of a hot spot beneath the SPT region [e.g., Howett et al., 2011] suggesting that the SPT may have a (partially) tectonic origin.

[4] Similarly, rift basins found on the Earth are generally complex features, but can be characterized by large-scale structural components, including: a depressed floor or trough, occasionally found to have relatively flat [e.g., Colman et al., 2003; Lagabrielle et al., 1997] and faulted [e.g., Allen and Allen, 2005; Withjack et al., 2002] floors; moderately-tosteeply dipping footwalls, uplifted flanks, and border faulting; and transform zones [Withjack et al., 2002]. Noting the topographic similarity between rift basins on the Earth and the SPT region of Enceladus, we seek to use simple terrestrially-motivated rift basin models to determine if the predicted magnitude of rift basin depression and mountainous uplift are consistent with observations. The models are intended to explain the overall shape of the region (i.e., floor depression and uplifted flanks) rather than the tiger stripe rifts themselves; our models seek to provide the tectonic setting in which such features could form (subject to additional tidal forcing, e.g., tidal flexure, differential rotation of the shell, etc.).

[5] To explore the possibility that similar processes formed the SPT, we examine three mechanical models that have historically been proposed to explain the topographic signatures observed in terrestrial rift basins, and examine which of these models are consistent with observed topographic features of the SPT. The models we examine here provide first order estimates of the processes controlling basin floor depth and the height of the rift wall bounding fault zone consistent with known material properties, rheological structure of the moon and observations. The models we use are summarized in the next sections, after which we present our results, and then compare the topography predicted by the models with observed topography at Enceladus.

\section{Mechanical Models of Basin Formation}

[6] The three terrestrial basin-forming models we consider are illustrated in Figure 2. The first model, formation due to thinning, shall describe the subsidence of the floor, while we intend to describe the flank uplift at the sides of the basin using the flexural models. These models are not necessarily independent and we later include the non-local flexural response of the ice into the thinning model. We describe the physical setting of each model in the following sections. We

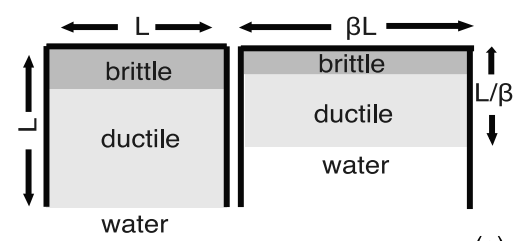

(a)
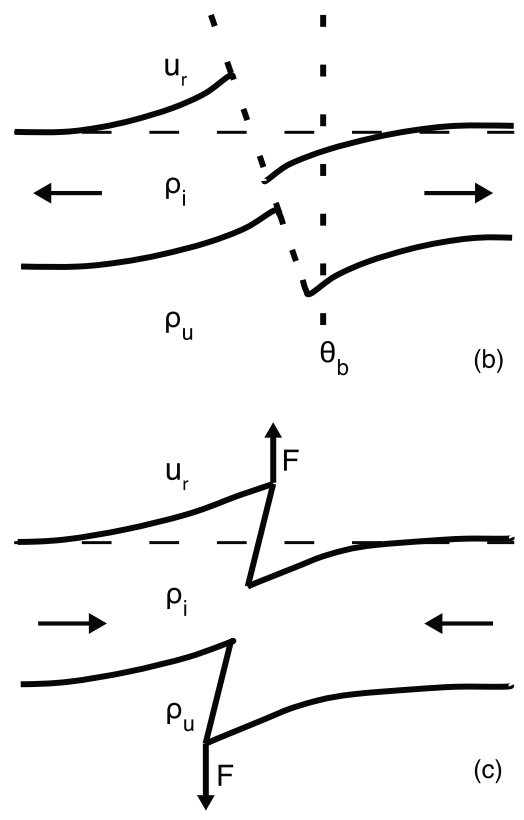

Figure 2. Schematics of the three basin models considered: (a) Thinning model: a section of crust is stretched by $\beta$, thereby thinning the solid layer by $\beta$, which leads isostatic imbalance and subsequent subsidence at the surface. (b) Extensional model of rift flank uplift: Dependent upon elastic response in the crust, we envision tensional forces pullapart a crustal block which breaks at angle $\theta_{b}$, causing uplift $u_{r}$. The footwall block (right) rises to maintain isostasy above the hanging wall block (left). (c) Compressional model of rift flank uplift: assuming an elastic response in the crust, we envision that horizontal forces push on a block until it breaks, causing a reverse fault and uplift $u_{r}$. All three models are dependent on layer properties, most importantly $\rho_{i}$ (ice density), and the density of the underlying substratum, $\rho_{u}$. In our models we consider several states for $\rho_{u}$. 


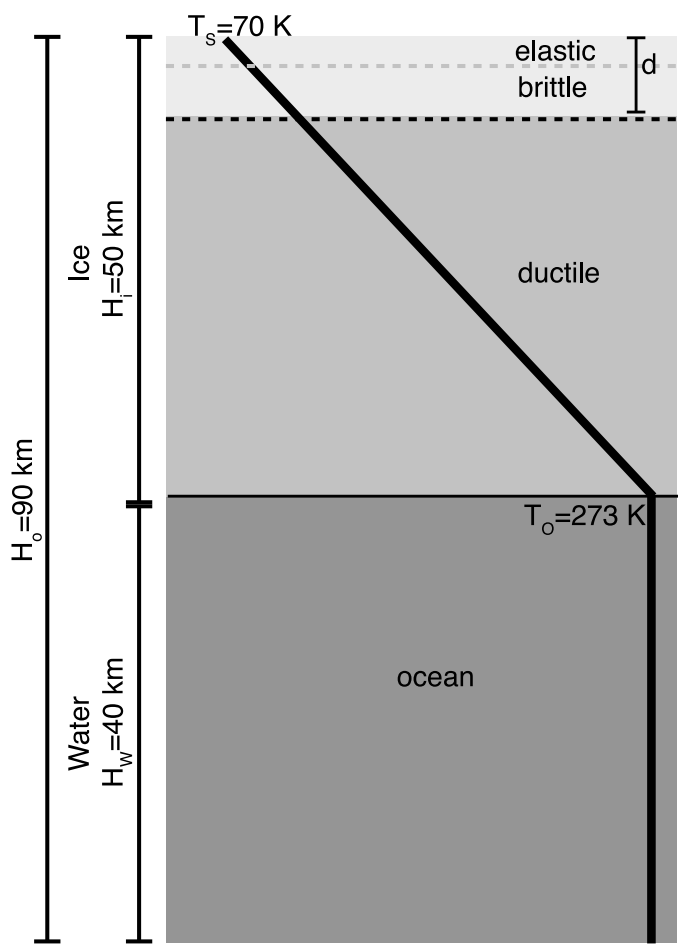

Figure 3. Model structure for Enceladus. We assume an ice shell of thickness $50 \mathrm{~km}$ over an ocean of $40 \mathrm{~km}$ depth, for a total outer water-ice layer of $90 \mathrm{~km}$. For models of flexure, we assume that the elastic thickness, $T_{e}$ (light gray), at Enceladus is $1 \mathrm{~km}$. The brittle layer of ice, or the depth $\mathrm{t}$ which fractures penetrate $(d)$, is taken to be $3 \mathrm{~km}$ (though we experiment with upper bounds in our results). We assume a surface temperature of $70 \mathrm{~K}$, a thermal gradient in the ice layer (thick black line), and an isothermal water ocean (dark gray) beneath at $273 \mathrm{~K}$.

note that Enceladus has a radius of just $250 \mathrm{~km}$, and so in contrast to the traditional Cartesian geometry often employed, our model calculations are carried out using a spherical geometry, described in Appendix A.

\subsection{An Icy Analog: Enceladus' Structure}

[7] Prior to constructing mechanical models of the SPT topography, we need to make assumptions about the internal structure and composition of Enceladus (Figure 3). We consider Enceladus to be differentiated with a rocky silicate core and a concentric water and ice layer of approximately $100 \mathrm{~km}$, up to Enceladus' effective radius of $250 \mathrm{~km}$. We assume this $100-\mathrm{km}$ outer layer consists of a liquid layer between 40 and $60 \mathrm{~km}$ thick (roughly consistent with estimates by Barr and McKinnon [2007], Barr [2008], Mitri and Showman [2008], Smith-Konter and Pappalardo [2008], Olgin et al. [2011], and Patthoff and Kattenhorn [2011]) beneath an upper layer of solid ice between 60 and $40 \mathrm{~km}$, respectively (consistent with estimates above). Unlike rocky material, ice remains brittle up to the melting point and so, consistent with previous studies, we define the brittle-ductile transition as the depth $d$ that a fracture penetrates. We generally assume the brittle layer thickness $H_{b}$ to be $3 \mathrm{~km}$, on the high-end but roughly consistent with Tobie et al. [2010], who showed the brittle-ductile transition depth to be $2-3 \mathrm{~km}$, but consider a range of fracture penetration depths. Later, we employ an elastic layer thickness to investigate the flexural response in the ice. Although not well constrained [Smith-Konter and Pappalardo, 2008], estimates for elastic thickness in the past range between $0.3 \mathrm{~km}$ in flexural studies by Giese et al. [2008], 0.4-1.4 km in heat flux analyses [Bland et al., 2007], at least $0.5 \mathrm{~km}$ in satellite reorientation models [Nimmo and Pappalardo, 2006], and up to approximately $8 \mathrm{~km}$ in tidal stress models [Smith-Konter and Pappalardo, 2008; Olgin et al., 2011]. In order to justify our own use of values, we computed preliminary results using a range of values for elastic and brittle thickness (between 1 and $10 \mathrm{~km}$ ), and compared these to the profile produced by Schenk and McKinnon [2009]. Keeping in mind that this single profile may not be completely representative, our best RMS misfit values come from elastic thicknesses between 1 and $5 \mathrm{~km}$; however, the elastic thickness of $1 \mathrm{~km}$ curve also reaches roughly the elevation that we would expect $(1 \mathrm{~km})$. It is due to this that we assume a $1 \mathrm{~km}$ elastic thickness, which also allows us to remain roughly consistent with the lower range of previous estimates.

\subsection{Subsidence of the SPT Floor}

[8] The first model we consider is motivated by early studies of sedimentary basins on Earth. McKenzie [1978] hypothesized that the stratigraphy of basins was formed through stretching and extensive normal faulting in the brittle crust (as suggested by Vening-Meinesz [1950]), followed by local isostatic subsidence. In contrast, for a basin to form without horizontal stretching, a large amount of material must be removed by erosion. In the case of Enceladus, even the most conservative estimate for elevation change would require the removal of $200 \mathrm{~m}$ [Thomas et al., 2007] of icy material across the approximately $300 \mathrm{~km}$ wide area across the South Pole. Although the terrestrial McKenzie [1978] model involves complexities due to thermal diffusion that are not relevant to our simpler ice-water structure, we postulate that the extensional portion of the model still provides a reasonable setting in which the SPT basin may have formed.

[9] The McKenzie [1978] thinning model (Figure 2a) describes a section of lithosphere that is stretched and thinned, allowing warmer subsurface material to rise to fill the thinned region from below. Neglecting elastic effects, the subsidence of the floor is described as the isostatic adjustment due to the thinning, where, as in Schenk and McKinnon [2009], the isostatic adjustment at the base of the shell is given by

$$
S_{i}=\frac{l \Delta \rho g_{b}}{\rho g_{s}}
$$

where $l$ is the amount of thinning of the ice (positive upwards from the base), $\Delta \rho$ is the density difference between ice and water at the base of the shell, $\rho$ is the ice density, and $g_{s}$ and $g_{b}$ are the surface and basal acceleration due to gravity, which ranges between 0.11 up to $0.15 \mathrm{~m} / \mathrm{s}^{2}$, respectively, depending on the thickness of the ice shell.

[10] This model is attractive in that it describes the formation of a depressed surface associated with a thinned ice layer. The model, however, has several deficiencies, including the possibility of regional (flexural) compensation. We can address this easily by including a finite elastic thickness of the ice. More problematic, we have employed 
this thinning model to describe the floor subsidence, but we also need to explain uplift at the boundary of the subsided region. To bound the magnitude of uplift, we imagine that we have one of two scenarios. In the first scenario, we assume the SPT is subject to a large-scale tensile stress and examine whether rift flank uplift as the result of the formation of extensional grabens is sufficient to explain the size of the mountains. In the second scenario, we imagine that the tensile stresses that caused subsidence are more local, allowing for extension in our basin that is then compensated by compressional stress at the edges of the region. These flexural models are described next.

\subsection{Flexural Response of the Ice Crust}

[11] We begin by describing flexure on a sphere [Turcotte et al., 1981]:

$$
D \nabla^{6} u_{r}+4 D \nabla^{4} u_{r}+E h R^{2} \nabla^{2} u_{r}+2 E h R^{2} u_{r}=R^{4}\left(\nabla^{2}+1-\nu\right) p
$$

where $p$ represents the pressure or force on the shell, $u_{r}$ is the resulting displacement in the radial dimension for which to solve, $R$ is the radius, $h$ is the elastic shell thickness, and $D$ is the flexural rigidity, defined as

$$
D=\frac{E h^{3}}{12\left(1-\nu^{2}\right)}
$$

with $E$ defined as Young's modulus and $\nu$ defined as Poisson's ratio. In contrast with previous studies, due to the fact that Enceladus' radius of curvature is so small, it is possible that the often-used Cartesian elastic plate model of lithospheric flexure [e.g., Turcotte and Schubert, 1982] may not be sufficiently accurate in predicting the flexure of Enceladus' ice crust and may have an effect on our tectonic problem [e.g., Yamaoka et al., 1986; Yamaoka, 1988; Mahadevan et al., 2010; Lagabrielle et al., 1997].

[12] Using a force-balance approach in the spherical dimensions, we use Legendre polynomials to determine the equation for the flexure of a spherical shell (approach is detailed in Appendix A), subject to line force $F_{r}$ as

$$
u_{r}=-\frac{R^{4}}{D} F_{r} \sum_{l=0}^{\infty} \frac{\left(l+\frac{1}{2}\right) \sin \phi_{0}}{G(l)} P_{l}\left(\cos \phi_{0}\right) P_{l}(\cos \phi)
$$

where $\phi_{0}$ is the colatitude angle at which the line force is applied (break point). We have also defined $G(l)$ here, as in Tanimoto [1997],

$$
\begin{aligned}
G(l)= & {\left[L^{2}-(1-\nu) L\right]\left(1-\frac{V_{l}}{U_{l}}\right)+12(1+\nu) \frac{R^{2}}{h^{2}} } \\
& \cdot\left(2-L \frac{V_{l}}{U_{l}}\right)+\frac{\Delta \rho g R^{4}}{D}
\end{aligned}
$$

where $L=l(l+1)(l$ is the angular degree), $\Delta \rho$ is the density contrast between the elastic layer and substratum, and $V_{l}$ and $U_{l}$ are Legendre coefficients defined in Appendix A. All calculations are carried to harmonic degree of 90 and solutions using a higher order do not alter results significantly. The line force $F_{r}$ to be applied in each case (thinning, extension, and compression) is derived in the following subsections.

\subsubsection{Flexural Response to Thinning}

[13] If we assume that at least the top layer of cold ice behaves elastically (elastic thickness: $1 \mathrm{~km}$ ) and that the extension is accommodated by a brittle layer $H_{b}$ of roughly $3 \mathrm{~km}$, the change in topography due to thinning at the SPT described above induces a flexural response in the elastic shell, described by the spherical flexure equation (equation (4)). The pressure at the base of the shell can be found as in Turcotte et al. [1981] for a change in topography:

$$
p=g\left[\rho_{i} h-\rho_{u} h_{g}-\left(\rho_{u}-\rho_{i}\right) u_{r}\right],
$$

Here, $\rho_{i}$ is the ice density and $\rho_{u}$ as the underlying substratum density (here defined as warm ductile ice), $h$ is the change in topography after thinning, $h_{g}$ is the displacement of the geoid, and $u_{r}$ is the flexure of the shell as before. The associated force over the basin $\left(F_{r}\right.$ in equation (4)) induces bending stress in the elastic shell in the $\phi$ direction (co-latitude), calculated as [Tanimoto, 1997]

$$
\sigma_{\phi \phi}=\frac{E}{1-\nu^{2}}\left(\varepsilon_{\phi \phi}+\nu \varepsilon_{\psi \psi}\right)
$$

where $\varepsilon_{\phi \phi}$ and $\varepsilon_{\psi \psi}$ are extensional strains. In determining stress over the shell, we can investigate points at which the yield strength of ice is surpassed, and therefore predict possible boundary fault locations (at which the uplift, calculated above, would occur). While the yield strength of ice at Enceladus is difficult to constrain [Rudolph and Manga, 2009], in this study we assume a yield strength of $1 \mathrm{MPa}$, consistent with terrestrial values [Bassis and Walker, 2011] and experimentally derived values for temperatures more appropriate for icy moons [e.g., Schulson, 1999].

\subsubsection{Boundary Flexure Due to Crustal Extension}

[14] It was suggested by Vening-Meinesz [1950] that if an extensional force were applied to a section of crust, normal faulting would eventually occur, illustrated in Figure 2b. In the past it has been applied to active rift systems as well as to basin settings; more specifically, to "syn-rift" basins that form in response to extension, e.g., seafloor spreading (a mechanism that had previously been suggested for the tiger stripes [e.g., Parkinson et al., 2008; Abramov and Spencer, 2009]). The Vening-Meinesz model considered the mechanics of blocks on either side of a fault (the original crustal block fractured along an angle, effectively leading to a reduction of mass of one crustal block (the footwall) and the addition of this mass to the opposite block (the hanging wall) [Watts, 2001, pp. 290-294], as illustrated in Figure 2b). In this terrestrial model, when considering the restoration of isostasy, the removal of mass from a section of crust will cause uplift and that area will rise; likewise the addition of mass to an adjacent section of crust, the hanging wall, will cause loading and sinking.

[15] In estimating the point of greatest curvature, and therefore bending stress, it is possible to determine the distance along the surface to a break in the plate. After this break, the gradual sinking of the downward block is eventually counteracted when the wedge is "caught" by the inward-sloping walls of the normal faults, unless extensional forces continue to act. This process produces a structure called a "graben," a bounding fault zone that characterizes a terrestrial basin [Schlische, 1991]. After Watts [2001], 


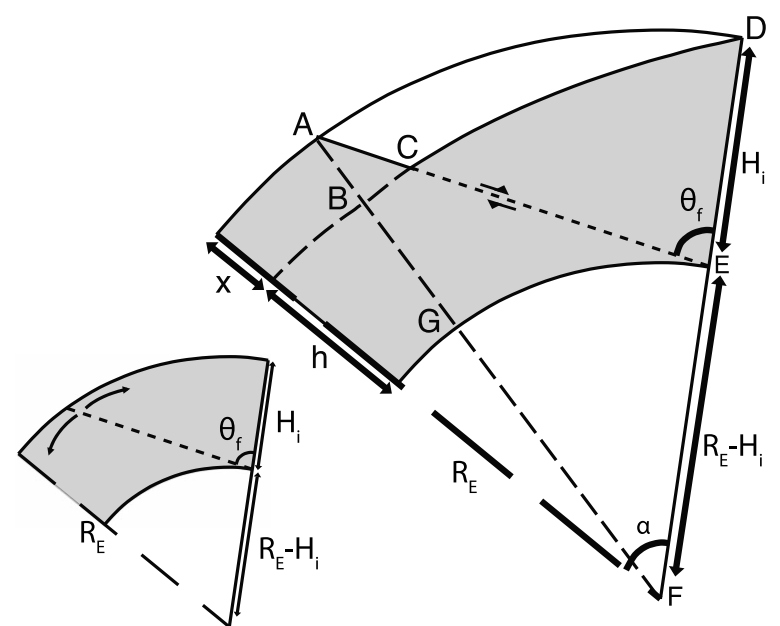

(a)

(b)

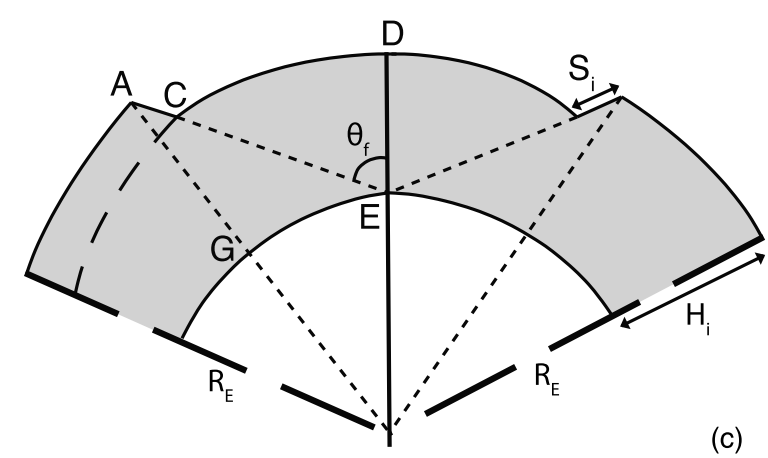

Figure 4. Schematic diagram illustrating the geometry of a section of shell in extension and the effective load necessary in order to return to isostatic equilibrium after a normal fault disrupts balance. (a) Prior to rifting, extensional stresses act on a section of shell. (b) The right hanging wall block slides downward, creating an isostatic imbalance thereby inducing uplift of the left footwall block. (c) A second break due to bending stress creates (ideally) a symmetric graben structure about the South Pole. Adapted to the spherical equivalent from Watts [2001, Figure 7.6].

faulting disturbs the isostatic balance of the initial block, and a load is applied by the crustal block on the left in Figure 4 onto the right block.

[16] While this is easily applied to the layer and density structure of the Earth, because the ice can remain brittle up to the melting point, in our study we can envision three different scenarios. In case A, we consider the (unrealistic) upper bound and imagine that brittle faulting due to extension penetrates the entire shell (fault depth $d=$ shell thickness $H_{i}$ ). In case $\mathrm{B}$, we allow faulting to penetrate a brittle layer thickness of $3 \mathrm{~km}$, overlying a ductile ice layer. In this case, we can assume that the ductile layer is either (i) entirely relaxed, thus negating any upward force at the base due to hydrostatic equilibrium, or (ii) that it is not entirely relaxed, and an upward force at its base due to the loading of the subsided block results in some amount of surface uplift. In case $\mathrm{C}$, if we assume that the ductile layer of ice is partially infiltrated by ocean water, then a dense layer underlies the surface ice and allows for a small amount of buoyant uplift.

[17] Regardless of the case we consider, in order to determine the flexure in equation (4), we must determine the force experienced by the shell in extension. To do this, we consider the process described by Watts [2001], and adapt it to our spherical frame (outlined in Appendix B). We find that the force on the shell at the plate break can be expressed as

$$
F_{E}=\frac{A_{A C D} \rho_{i} g S_{i}}{T_{e} L_{\phi}}
$$

where we have defined $T_{e}$ as the elastic thickness; $\rho_{i}$ as the density of ice (averaged); $g$ is the acceleration due to gravity; $S_{i}$ is the vertical distance the subsided block has been forced down as defined in equation (1); $L_{\phi}$ is the length of the bending surface between breaks in the $\phi$ (co-latitude) direction, here taken to be the width of the SPT; and $A_{A C D}$ is the area of semi-triangle ACD in Figure 4. This equation effectively calculates the force initiated by the subsided block that impinges upon the base of the shell at the opposite block and can be simplified down to the linear elastic plate case found in Watts [2001] by altering the geometry in Figure 4.

\subsubsection{Boundary Flexure Due to Crustal Compression}

[18] Bullard [1936] hypothesized instead that rift basins formed in compressional stress regimes. We suppose that local extension that created the subsided floor of the basin may have resulted in corresponding compression at the border of the region that then results in uplift. In the Bullard [1936] theory, compression would cause a reverse fault, allowing one crustal block to ride up on the opposing block, resulting in buoyancy-induced flexure (dependent on density differences between layers), as illustrated in Figure 2c. Bullard [1936] further proposed that the reverse faulting causes bending of the opposing block, leading to a second plate break (from extension) in the plate where the bending stress is greatest, allowing the central block to sink, thereby forming a basin. To determine the depth and width of a rift valley in this model, Bullard used an early broken elastic plate model developed by Jeffreys [1915], using a calculated force on the end of the plate.

[19] As in the extensional case (section 2.3.2), while this proposed process works well for the structure and densities of the Earth's layers, we have to think carefully about how to apply an analogous model to the ice and water layers at Enceladus. In case A, we imagine again that the entire ice shell behaves as a brittle layer, allowing faults to penetrate through to the base $\left(d=H_{i}\right)$; in case $\mathrm{B}$, we assume a more realistic brittle layer thickness of $3 \mathrm{~km}$, allowing fractures to penetrate to this depth only over a ductile ice layer below; and in case $\mathrm{C}$, we again investigate the possibility that there exists an ice-water mixed layer that allows for buoyant uplift of the dense, cold surface ice. Of course, as before, the outcome is dependent upon the density differences between layers; by splitting Case B into two subsets (density difference between warm and cold ice vs no density difference at all), we will show that in this case that densities have less of an effect, ceding dominance to the assumed elastic thickness term $(1 \mathrm{~km})$.

[20] As in the extensional model, we use our spherical flexure equation (equation (4)) and apply a calculated line 


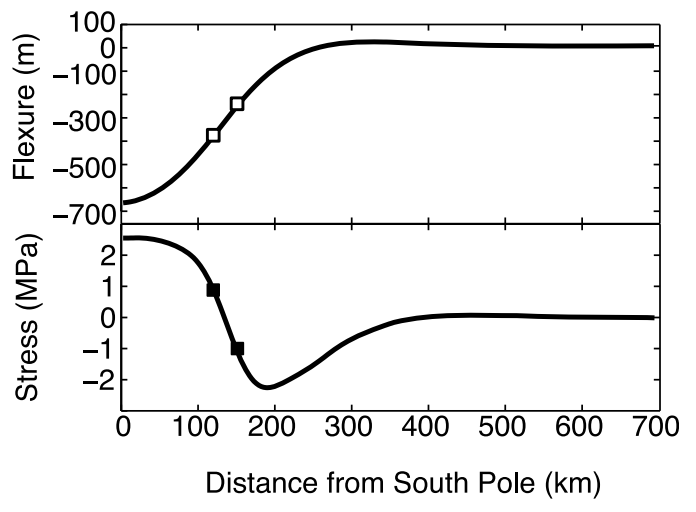

Figure 5. (top) Flexure of the ice shell due to thinninginduced subsidence, used to calculate (bottom) associated tensional stress. Black squares in Figure 5, bottom, show points at which stress surpasses $1 \mathrm{MPa}$, our assumed yield strength of ice. These locations are translated to Figure 5, top, in white, showing the locations at which the ice will fail, noting that negative stress implies compressive stress. This break allows for uplift at the boundary and shows the width of our predicted basin, roughly 240 to $310 \mathrm{~km}$ across, assuming an elastic response in the ice.

force at the plate break to determine uplift. To determine this upward force acting at the plate break, we adapt the approach of Bullard [1936] (as adapted by Watts [2001]) to our spherical structure, and express the force as

$$
F_{C}=\frac{\rho_{u} L_{\phi} g x}{2 T_{e}}
$$

where $\rho_{u}$ is defined again here as the underlying layer density, dependent on which case (A, B, or C) is being considered (ice or water density). This force is then applied at the plate break to determine uplift.

[21] Geological evidence has subsequently shown that most terrestrial rift basins formed in extensional rather than compressional tectonic stress regimes, a criticism that has since proven fatal to the Bullard [1936] hypothesis [e.g., [Weissel and Karner, 1989; Watts, 2001], as measurements of gravity and increased observations of rift valleys could be better explained in an extensional regime. Nonetheless, compression has been proposed as a mechanism for the SPT mountainous features and we consider this as a viable model for the uplift surrounding the SPT basin, even if it is not observed on Earth.

\section{Model Results and Comparison to Topographic Observations}

\subsection{Subsidence of the Basin Floor Due to Thinning}

[22] In applying our thinning model (i.e., section 2.2), we assume that a section of the icy shell was stretched by a factor of $\beta$ due to far-field extensional stresses [e.g., Withjack et al., 2002; Allen and Allen, 2005] leading to thinning of the ice shell in the localized region beneath the SPT (final extended basin width can be calculated by $\beta$ and an initial basin width). In this case, this assumed instantaneous thinning causes subsidence $S_{i}$ (equation (1)). We find that by stretching the region by $\beta=1.11$ to 1.13 thins the crust by approximately 5 to $6 \mathrm{~km}$, and the resulting topographic subsidence is approximately $600 \mathrm{~m}$. This value corresponds reasonably well with previously published estimates of SPT topography and the elevation change, which range between 200 and $800 \mathrm{~m}$ [Thomas et al., 2007; Roberts and Nimmo, 2008; Schenk and McKinnon, 2009; Helfenstein et al., 2011].

\subsection{Flexural Response of the Crust}

\subsubsection{Flexural Response to Thinning}

[23] The change in topography due to thinning at the SPT induces a flexural response in the shell, described by equation (4). This flexure (Figure 5, top) and stress (Figure 5, bottom) are shown in Figure 5. Black squares on the stress curve denote the location at which the stress in the elastic shell surpasses $1 \mathrm{MPa}$, our projected yield strength of ice. These points result in a basin floor with a diameter of approximately $250-310 \mathrm{~km}$, illustrated by white squares in Figure 5 (top). This result roughly agrees with observations of the basin width (approximately $300 \mathrm{~km}$ across in the $\phi$ direction).

\subsubsection{Boundary Zone Uplift by Extensional Forces}

[24] Porco et al. [2006] observe that the mountainous boundary reaches approximately $1 \mathrm{~km}$ in height, a value matched by Schenk and McKinnon [2009]. In applying the model of crustal extension attributed to Vening-Meinesz [1950] (section 2.3.2), we can calculate the amount of flexural footwall uplift, dependent upon the normal faulting angle [Watts, 2001] at the break. Figure 6 shows the amount of uplift for an assumed fault angle of $20^{\circ}$ and floor depression (about $500 \mathrm{~m}$ ), for an elastic thickness of $1 \mathrm{~km}$. The value is similar to best-fit values used to assess normal faulting on Europa, an icy satellite of Jupiter with a possibly similar ice shell. Nimmo and Schenk [2006] studied two possible normal faults on Europa and found best-fit angles to be $11^{\circ}$ and $22^{\circ}$. Figure 6 shows that for this faulting angle

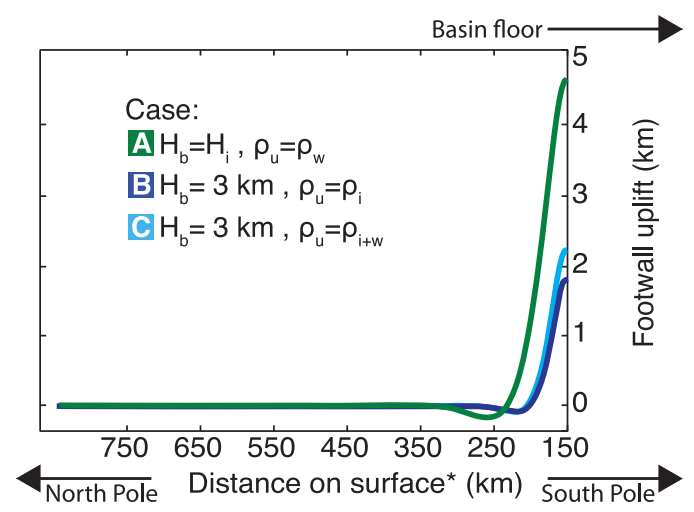

Figure 6. Footwall uplift in the case of extensional forces at the boundary of the SPT area. Uplift varies with assumed layer properties. Case A (green): Fracturing penetrates the entire ice thickness $\left(d=H_{i}\right)$, and underlying substratum taken to be water $\left(\rho_{u}=\rho_{w}\right)$. Case B (dark blue): Fracturing penetrates an brittle layer of $3 \mathrm{~km}$, over a warm ductile ice layer, whose density is related to the temperature gradient in the ice layer (coldest ice at surface: $933 \mathrm{~kg} / \mathrm{m}^{3}$, warmest

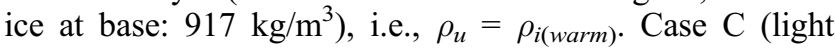
blue): Fracturing penetrates a brittle layer of $3 \mathrm{~km}$, over a substrate of a "slushy" ice-water mixture. 


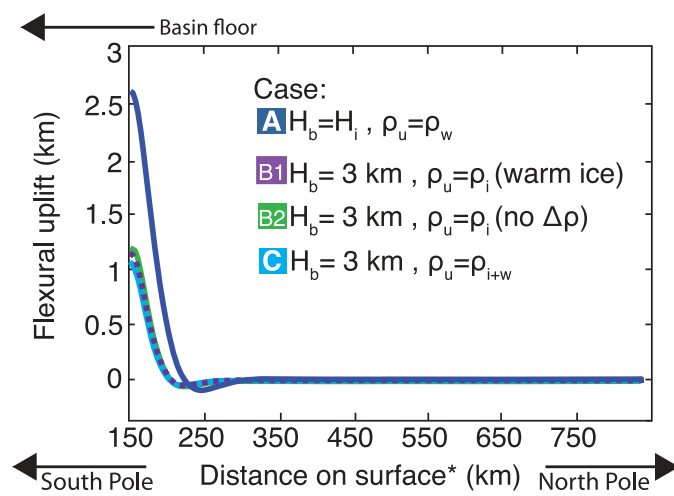

Figure 7. Footwall uplift profile in the case of compressional forces at the boundary of the SPT area. Case A (dark blue): Fracturing penetrates the entire ice thickness $\left(d=H_{b}=\right.$ $\left.H_{i}\right)$, and underlying substratum taken to be water $\left(\rho_{u}=\rho_{w}\right)$. Case B1 (purple): Fracturing penetrates a brittle layer of 3 $\mathrm{km}$, over a warm ductile ice layer, whose density is related to the temperature gradient in the ice layer (coldest ice at surface: $933 \mathrm{~kg} / \mathrm{m}^{3}$, warmest ice at base: $\left.917 \mathrm{~kg} / \mathrm{m}^{3}\right)$, i.e., $\rho_{u}=$ $\rho_{i(\text { warm) }}$. Case B2 (green): similar to Case B1 in structure, this case was used to illustrate the small effect of assumed substratum density on result. In this case, we assume no density difference between the brittle ice and underlying ice, resulting in only a small change from the density-gradient version in Case B1. Case C (light blue): Fracturing penetrates a brittle layer of $3 \mathrm{~km}$, over a substrate of a "slushy" ice-water mixture.

in cases $\mathrm{A}, \mathrm{B}$, and $\mathrm{C}$, if the south polar basin formed in this extensional manner, flexural uplift at the boundary is predicted to be approximately $4.6 \mathrm{~km}$ in case $\mathrm{A}\left(d=H_{b}=H_{i}\right)$, $1.8 \mathrm{~km}$ in case $\mathrm{B}\left(d=H_{b}=3 \mathrm{~km}\right)$ assuming the ice below is not completely relaxed (total viscous relaxation results in sinking rather than uplift), and $2.05 \mathrm{~km}$ in case $\mathrm{C}\left(d=T_{e}\right)$ over a "slushy" ice-water layer. Even this lowest result (Case B, also the most realistic) is about twice the height of the uplift observed today. Though it is likely that, over its active lifetime, the SPT has undergone additional topography-altering events (i.e., heights predicted here may have been altered by subsequent tectonic events), the requirements (i.e., cases A, B, and C) for uplift under extension are unlikely. So we conclude that the extension model cannot realistically predict the basin at the South Pole.

3.2.3. Boundary Zone Uplift by Compressional Forces

[25] Similarly, in applying the compressional uplift model as described by Bullard [1936] (section 2.3.3), we find that uplift can develop at the edge of the region if we assume that compression is accommodated at that location in response to the extension of the floor. We find that the footwall uplift height of a basin with floor depth of roughly $500 \mathrm{~m}$ and elastic thickness of $1 \mathrm{~km}$ reaches approximately $2.6 \mathrm{~km}$ in case A $\left(d=H_{b}=H_{i}\right)$, approximately $1.15 \mathrm{~km}$ in case B $\left(d=H_{b}=3 \mathrm{~km}\right)$ in which the brittle layer overlies warmer ductile ice, and roughly $1.06 \mathrm{~km}$ in case $\mathrm{C}(d=3$ $\mathrm{km}$ over "slushy" water-ice matrix). The most realistic scenario, case B (in which the brittle layer accommodates fracture overlying warmer ductile ice) also gives promising results, approximately the mountain height observed today
$(1 \mathrm{~km})$. These results are shown in Figure 7 and further discussed in our integrated model in the following section.

\section{Discussion}

\subsection{Thinning and Flexural Uplift: Integrated Model}

[26] In our best integrated model from those analyzed in the previous section, we postulate that the ice shell at the South Pole was stretched by local extensional stresses, which induced thinning of the shell. The associated subsidence, as described by our thinning model, places the SPT floor in a depression of $600 \mathrm{~m}$. The local extension of the area is compensated by compression at the boundary of the thinned region, resulting in reverse faulting of the brittle ice layer (of thickness $3 \mathrm{~km}$ ) overlying a warmer ductile ice layer, which results in uplift of roughly $1.15 \mathrm{~km}$. We postulate that the overall bowl-shape of the SPT region is reflected in this model and that it begets generally favorable agreement with past observations of topography there. This integrated model also assumes a structure similar to that suggested by past analyses (e.g., Smith-Konter and Pappalardo [2008], Patthoff and Kattenhorn [2011], and others), with an ocean layer of $40 \mathrm{~km}$ underlying a solid ice shell of $50 \mathrm{~km}$, which features an elastic thickness of $1 \mathrm{~km}$. The stresses that we predict in the shell are larger than diurnal tidal stresses at Enceladus [Rudolph and Manga, 2009] but on the order of previous estimates of tectonic stresses [e.g., Nimmo and Pappalardo, 2006] and estimates of stress due to thickness changes [Manga and Wang, 2007]. The locations along the surface where the stress surpasses our assumed yield strength of $1 \mathrm{MPa}$ (also marked in Figure 5) are marked in Figure 8 as magenta (tensile) and orange (compressive) squares. It is worthwhile to note that the maximum compressive stress occurs at the flank location, bolstering our argument that our compressive model of flank uplift is appropriate here. It is also relevant to point out that, while our model exhibits axial symmetry, the "tiger stripes" notably break that symmetry, suggesting that the additional processes, such as tidal stresses, may be responsible for the observed symmetry-breaking formation of these features; the tectonic stress and thinning provide the background environment which makes this likely.

[27] Figure 8 shows the topography derived by Schenk and McKinnon [2009] through the use of digital terrain maps, illustrating the bowl-shaped basin of the SPT and its boundary uplift relative to sea level. As many properties and parameters at Enceladus are poorly constrained, the gray block in Figure 8 exhibits the effect on the results when model parameters are varied. Varying the thinning model between upper and lower limits of published parameters at Enceladus cause the margin of resulting subsidence levels (gray) to remain fairly wide. Elevation estimates from published sources mentioned above are shown on the plot in Figure 8 in varying colors, and exhibit a good fit within the grayed area of our model estimates. This agreement with previously published values reinforces the possibility that the extensional model is a viable model for subsidence of the floor (and therefore basin formation) at the SPT region.

[28] While we include simplifying assumptions in our models (e.g., layer composition, column-averaged densities, assumed elastic thickness), our basin models do, in most cases, reasonably describe the subsidence and mountainous 


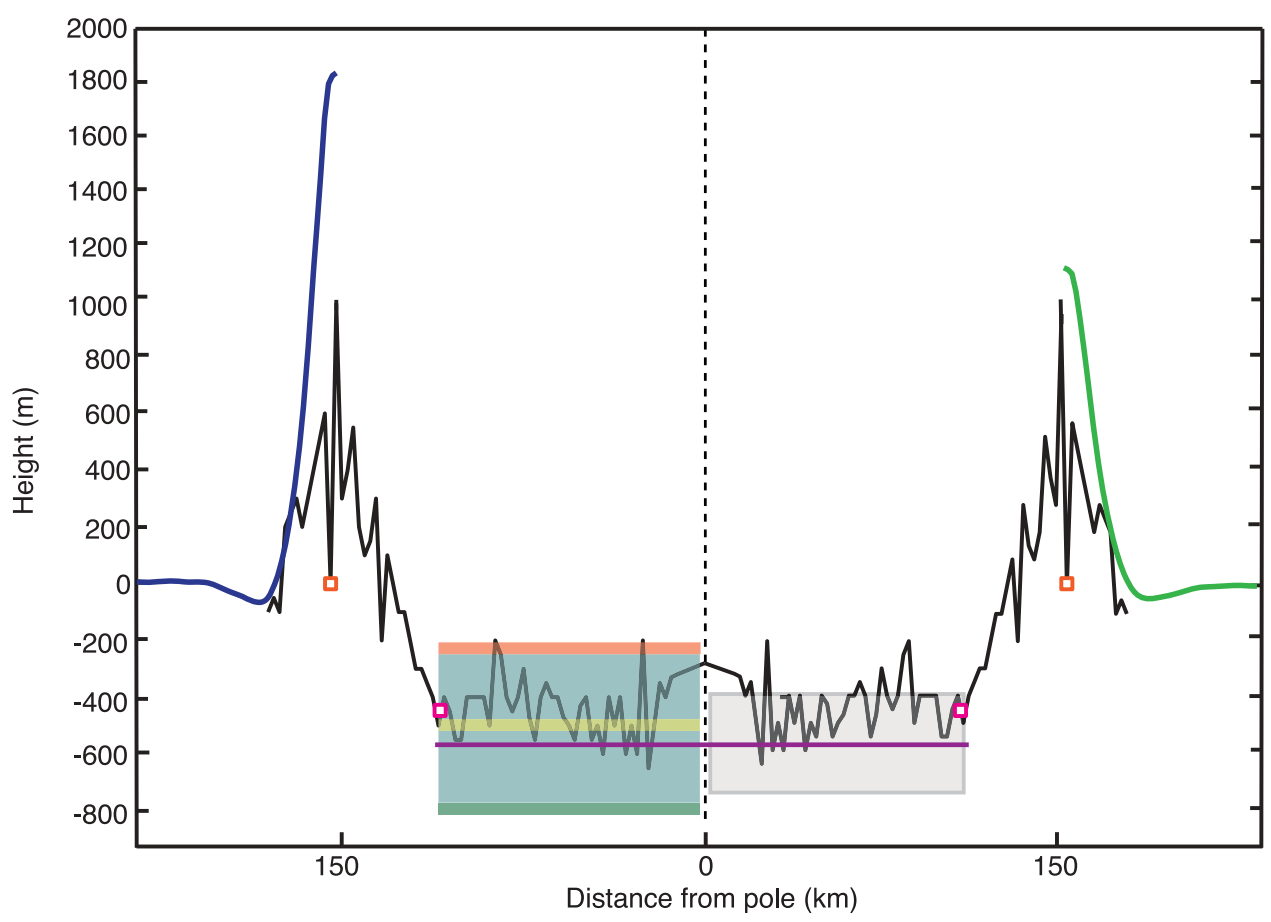

Figure 8. The topographic profile (black) of the SPT from Schenk and McKinnon [2009] is used to illustrate model results. Left hand navy blue line denotes the best-fitting result from the extensional model. This reaches approximately $1.8 \mathrm{~km}$ in height, assuming a brittle layer overlying a warm ductile ice layer. On the right hand side, the green line denotes our best fit curve of flank uplift in the compressional model, reaching $1.15 \mathrm{~km}$, assuming a brittle layer of $3 \mathrm{~km}$ over a ductile layer of ice beneath. This result and structure match previous estimates of elevation and internal structure. Purple line denotes the subsidence we find from our thinning model ( $600 \mathrm{~m}$ depression); we put this in context of previous estimates of the depression depth at the SPT (Thomas et al. [2007] and Roberts and Nimmo [2008] (red); Helfenstein et al. [2011] (yellow); and Schenk and McKinnon [2009] (green (lowest estimate)); teal box denotes the span of estimated depths at the South Pole). Due to the uncertainty in parameters at Enceladus, we include error in our assessment, marked by the gray box. Maximum extensional stress (magenta) and compressional stress (orange) are marked by boxes (see Figure 5). Note that the profile is a single side of the basin mirrored over the central axis.

uplift at the boundary and provide self-consistent estimates of the uplift and subsidence of the topography.

\subsection{Terrestrial Basins and Implications for the SPT}

[29] Standard rift basins in the terrestrial setting are related to stresses in the crust and are idealized as manifestations of active or passive rifting. During active rifting, deformation is associated with impingement by a mantle plume (warm material) on the base of the lithosphere, causing thinning, doming, and eventual downwarping of the area, along with extensional rifting. Alternatively, under passive rifting, farfield tensional strains in the lithosphere cause a weakening of the surface material, allowing for upwelling of hot material [e.g., Huismans et al., 2001]. Most terrestrial basins exhibit features of both behaviors [Allen and Allen, 2005], and so these are generally regarded as end-members of the formation spectrum. They mostly refer to whether or not a basin forms at a plate boundary (active) or not (passive). Rift basins in general are complex features, but can be characterized by large-scale structural components, including: a depressed floor or trough, occasionally found to be relatively flat [e.g., Colman et al., 2003; Lagabrielle et al., 1997] and faulted [e.g., Allen and Allen, 2005; Withjack et al., 2002]; moderately-to-steeply dipping footwalls, uplifted flanks, and border faulting; and transform zones [Withjack et al., 2002]. These large-scale components of basin topography are observed at the SPT of Enceladus - the circular depression features a rifted floor, steep cliff footwalls, and uplifted boundary flanks (see original DEM profile by Schenk and McKinnon [2009]). The SPT also overlies a high-heat temperature anomaly, suggestive of the subsurface heating that normally goes along with basin formation, as noted above. The possibility that basin-forming mechanisms may be used to explain the formation of circular depressions on planetary surfaces has been suggested previously, both on Earth (e.g., the Michigan Basin [Sleep et al., 1980]), and on other planets (e.g., Atalanta Planitia [Solomon et al., 1982], and regions of Atropos Tessera [Ori and Baker, 1995], both on Venus) to explain the formation of circular basins that did not originate via impact cratering.

[30] An example of thermally-driven basin formation presents itself in the North Fiji Basin (NFB) in the Pacific Ocean. Overlying a hot spot, Lagabrielle et al. [1997] showed that active upper mantle convection is a key process in controlling the crustal dynamics of the NFB, and is largely independent from the evolution of larger structural 
features, such as the Vitiaz and New Hebrides subduction zones [Lagabrielle et al., 1997]. This is an important point in our Enceladus application: the spreading at the ridges is largely controlled by the existence of subsurface heat and subsequent convection rather than the action of subduction zones at the borders. This independence of the NFB basin formation from an adjacent subduction zone suggests that the maintenance and propagation of its ridges may be similar to that of the SPT, as the SPT features are likely dependent upon subsurface processes related to south polar hot spot rather than active subduction, i.e., heat and/or subsurface convection [Barr, 2008; Mitri and Showman, 2008] may have driven (and continue to drive) the evolution of the SPT. This study shows that the depression at the SPT could have formed during a tectonic event analogous to those responsible for the formation of rift basins on our own planet, an inquiry motivated by our simple observation that the NFB seemed to be somewhat similar in appearance and thermal setting. While current surface expressions of stress and activity (including the tiger stripes) are likely just the latest in a long history of deformation, we suggest that the original subsidence of the SPT floor, and therefore the largescale shape of the region still seen today, may have originated in a similar fashion to Earth's basins.

[31] It is also notable that stresses computed due to a change in topography of the icy shell are on the order of estimates of tidal stresses, bolstering the possibility that, first, stresses on this order exist in the shell, and second, that the shell reacts to such stresses (e.g., the activation of the tiger stripes). Our hypothesis was motivated by the simple observation that terrestrial rift basins have similar structural characteristics, including those mentioned above and an associated "starfish" pattern at the tectonic boundary, seen both at the SPT and tectonic boundaries on Earth [Mahadevan et al., 2010].

\section{Conclusions}

[32] Through an application of simple terrestrial basin models, we conclude that it is possible that the SPT of Enceladus formed by processes analogous to those associated with the formation of our own terrestrial rift basins. This application was motivated by the observation that the SPT topography agrees with the overall characteristics of a terrestrial rift basin, exhibiting a rifted central depression, steep footwalls, uplifted flanks, and border faulting, in addition to its existence over a high-heat spot that is independent of active subduction zones. Our best integrated model, assuming local extension of the shell at the South Pole that is accommodated by compression at the border, shows that our SPT basin attains a shape that approximates previous topographic estimates to a fair degree. Although we speculate on both compressive and extensional scenarios, in reality the models constitute end-members of a continuous spectrum of processes; it is widely thought that Enceladus' SPT current form is the result of a sequence of tectonic events that continues today, and is likely a combination of many processes. Here we speculate only on the origin of the depression and ringed boundary uplift region.

[33] While the compression case showed more favorable results, it is likely that the elevation of the mountains, in either case, has been modified by subsequent tectonic events. Our results, while based on simple models, lend themselves to the possibility that the general shallow bowl-shape of the SPT may have an origin similar to that of terrestrial rift basins. Future work could include enhanced modeling, most specifically the use of 3D geometries, the influence of rheology, and better estimates of ice strength at the SPT. Enhancing this model to the level of current advanced terrestrial basin models (e.g., those used for petroleum geology) would be a prudent next step.

\section{Appendix A: Flexure of the Spherical Shell}

[34] Idealizing Enceladus as a spherical shell, the flexure equation on a sphere can be written as the sixth-degree partial differential equation [Turcotte et al., 1981]

$$
D \nabla^{6} u_{r}+4 D \nabla^{4} u_{r}+E h R^{2} \nabla^{2} u_{r}+2 E h R^{2} u_{r}=R^{4}\left(\nabla^{2}+1-\nu\right) p
$$

where $h$ is the thickness of the shell, $R$ is the radius of curvature, $E$ is Young's modulus, $\nu$ is Poisson's ratio, and where $D$ is the flexural rigidity, defined as

$$
D=\frac{E h^{3}}{12\left(1-\nu^{2}\right)}
$$

Equation (2) can alternately be written [Beuthe, 2008; Sandwell and Schubert, 2010] as

$$
\begin{gathered}
\eta D \nabla^{2} \nabla^{\prime 2} \nabla^{\prime 2} u_{r}+F R^{2} \nabla^{\prime 2} \nabla^{2} u_{r}+E h R^{2} \nabla^{2} u_{r}+R^{4}\left(\nabla^{2}+1-\nu\right) \\
\cdot \Delta \rho g u_{r}=R^{4}\left(\nabla^{2}+1-\nu\right) q_{0} \partial(\phi)
\end{gathered}
$$

where $F$ is the end load on the shell, $q_{0}$ is the point vertical load applied at $\phi$, and $\phi$ is the polar angle (or co-latitude). We define $\eta$ as in Sandwell and Schubert [2010] as

$$
\eta=\frac{12 R^{2}}{12 R^{2}+h^{2}}
$$

In equation (A2), the differential operators $\nabla^{2}$ and $\nabla^{\prime 2}$ are given by

and

$$
\nabla^{2}=\frac{1}{\sin \phi} \frac{\partial}{\partial \phi}\left(\sin \phi \frac{\partial a}{\partial \phi}\right)
$$

$$
\nabla^{\prime 2}=\nabla^{2}+2 a
$$

where $a$, in both cases, serves as a placeholder for the subject of the operator. Beuthe [2008] shows that these equations stem from a balance of forces. Assuming a symmetric shell as in Tanimoto [1998], we can define a balance of forces in spherical coordinates as

$$
\begin{gathered}
\frac{1}{\sin \phi} \frac{\partial}{\partial \phi}\left(\sin \phi \bar{\sigma}_{r \phi}\right)-\bar{\sigma}_{\phi \phi}-\bar{\sigma}_{\psi \psi}=-\frac{R}{h} f_{r}+\frac{R}{h} \Delta \rho g u_{r} \\
\frac{1}{\sin \phi} \frac{\partial}{\partial \phi}\left(\sin \phi \bar{\sigma}_{\phi \phi}\right)+\bar{\sigma}_{r \phi}-\bar{\sigma}_{\psi \psi} \cot \phi=0 \\
\frac{1}{\sin \phi} \frac{\partial}{\partial \phi}\left(\sin \phi M_{\phi \phi}\right)+R h \bar{\sigma}_{r \phi}-M_{\psi \psi} \cot \phi=0
\end{gathered}
$$


with $\phi$ the polar angle (or colatitude) as above and $\psi$ the azimuthal angle (or longitude) and $f_{r}$ is the vertical line load acting at $\phi=\phi_{0}$. Equations (A7), (A8), and (A9) represent the force balance in $r, \phi$, and moment balance about a constant colatitude [Timoshenko and Woinosky-Krieger, 1959; Tanimoto, 1997], respectively. $\bar{\sigma}$ represents the depthaveraged stress over the shell thickness. $u_{r}$ is the vertical displacement in the radial direction (deflection) as defined previously in the main text. As we will simplify our problem by ignoring azimuthal variations, then from Beuthe [2008], the stress component can be written

$$
\bar{\sigma}_{\phi \phi}=\frac{E}{1-\nu^{2}}\left(\varepsilon_{\phi \phi}+\nu \varepsilon_{\psi \psi}\right)
$$

where $\varepsilon$ is the extensional strain. This can be related to the displacement as

$$
\varepsilon_{\phi \phi}=\frac{u_{r}}{R}+\frac{1}{R} \frac{\partial u_{\phi}}{\partial \phi}
$$

and

$$
\varepsilon_{\psi \psi}=\frac{u_{r}}{R}+\frac{\cot \phi}{R} u_{\phi}
$$

The bending moments $M_{\phi \phi}$ and $M_{\psi \psi}$ are related to the curvature of the shell and can also be expressed in terms of the displacement.

$$
M_{\phi \phi}=\frac{D}{R^{2}}\left[\left(\frac{\partial^{2} u_{r}}{\partial \phi^{2}}-\frac{\partial u_{\phi}}{\partial \phi}\right)+\nu\left(\left(\frac{\partial u_{r}}{\partial \phi}-u_{\phi}\right) \cot \phi\right)\right]
$$

and

$$
M_{\psi \psi}=\frac{D}{R^{2}}\left[\left(\left(\frac{\partial u_{r}}{\partial \phi}-u_{\phi}\right) \cot \phi\right)+\nu\left(\frac{\partial^{2} u_{r}}{\partial \phi^{2}}-\frac{\partial u_{\phi}}{\partial \phi}\right)\right]
$$

Since the vertical displacement that we seek should be of the spheroid vector form, then we can expect it to have the form [Tanimoto, 1997; Sandwell and Schubert, 2010]

$$
u_{r}=\sum_{l=0}^{\infty} U_{l} P_{l}(\cos \phi)
$$

and

$$
u_{\phi}=\sum_{l=1}^{\infty} V_{l} \frac{d P_{l}}{d \phi}
$$

Employing (for now) the asymptotic solution found in Tanimoto [1997], assuming that a downward line force loads the shell at $\phi=\phi_{0}$ (axisymmetric), we can write the full solution for the vertical deformation $u_{r}$ using the asymptotic formula for Legendre polynomials as

$$
\begin{aligned}
u_{r}= & -A\left(\frac{\sin \phi_{0}}{\sin \phi}\right)^{1 / 2} \exp \left(-\alpha\left(\phi-\phi_{0}\right)\right) x\left(\cos \alpha\left(\phi-\phi_{0}\right)\right. \\
& \left.+\sin \alpha\left(\phi-\phi_{0}\right)\right)
\end{aligned}
$$

Here, $A$ is a conglomerate of parameters:

$$
A=\frac{R^{4}}{2 \sqrt[2]{2} D k^{3}} F_{r}
$$

and $\alpha$ can be expressed as $\frac{k}{\sqrt[2]{2}}$. In these expressions, $k$ is defined as [Tanimoto, 1998]

$$
k^{4}=\frac{12\left(1-\nu^{2}\right) R^{2}}{h^{2}}\left(1+B_{E}\right)
$$

where $B_{E}$ represents the buoyancy ratio between the elastic crust and the viscous layer below it:

$$
B_{E}=\frac{\Delta \rho g h}{E}\left(\frac{R}{h}\right)^{2}
$$

More fully, we can also write the complete solution using Legendre Polynomials as

$$
u_{r}=-\frac{R^{4}}{D} F_{r} \sum_{l=0}^{\infty} \frac{\left(l+\frac{1}{2}\right) \sin \phi_{0}}{G(l)} P_{l}\left(\cos \phi_{0}\right) P_{l}(\cos \phi)
$$

Here, we have defined $G(l)$ as in Tanimoto [1997]:

$$
\begin{aligned}
G(l)= & {\left[L^{2}-(1-\nu) L\right]\left(1-\frac{V_{l}}{U_{l}}\right)+12(1+\nu) \frac{R^{2}}{h^{2}}\left(2-L \frac{V_{l}}{U_{l}}\right) } \\
& +\frac{\Delta \rho g R^{4}}{D}
\end{aligned}
$$

where $V_{l}$ and $U_{l}$ are Legendre coefficients and their ratio can be simplified, as shown in Tanimoto [1997], and written

$$
\frac{U_{l}}{V_{l}} \approx \frac{l(l+1)}{(1+\nu)}
$$

Applying this flexural model to Enceladus' ice crust requires specification of various ice parameters as well as the magnitude and nature of the deforming load $\left(F_{r}\right)$ applied to the shell. In each of the three basin models used in this study, $F_{r}$ will be defined as a load in the radial direction that impinges on the shell. Using the flexure to determine the stress within the shell, it is possible to determine the point at which the shell is likely to break (using an assumed value for the strength of ice).

\section{Appendix B: Cartesian Geometry Results and Comparison}

[35] Enceladus has a small radius of curvature, and we therefore adopted a spherical geometry with which the deformation was modeled. In the interest of comparison, we present the Cartesian results of the models described in the text.

[36] In the application of the compressional model of Bullard [1936], we find flexure $y$ as a function of distance $x$ away from the plate break using the broken elastic plate solution to find a maximum uplift of roughly $0.5 \mathrm{~km}$. This an order of magnitude less than our results (computed in a spherical geometry) of $1.15 \mathrm{~km}$. It could be that the difference can be attributed to stresses due to the curvature of the shell adding to the compressive force in the spherical model. 
[37] In the case of the extension model of Vening-Meinesz [1950], we use the Cartesian geometrical set-up by Watts [2001], which we had adapted to spherical form, to determine the uplift by finding the force on the block and applying it to the end of a broken plate. In this case, we find a total uplift, assuming a $20^{\circ}$ fracture, of approximately $0.6 \mathrm{~km}$. This is about one-third of our lowest results in the spherical formulation, which is to be expected from our geometric approach. The difference between flexure results likely arises from the application of the spherical flexure formula which incorporates stresses due to the curvature of the shell into the results.

[38] Acknowledgments. This work was partially supported by the Michigan Space Grant Consortium. We also thank the Associate Editor and two anonymous reviewers, whose comments and suggestions substantially improved the manuscript.

\section{References}

Abramov, O., and J. R. Spencer (2009), Endogenic heat from Enceladus' south polar fractures: New observations, and models of conductive surface heating, Icarus, 199, 189-196, doi:10.1016/j.icarus.2008.07.016.

Allen, P. A., and J. R. Allen (2005), Basin Analysis: Principles and Applications, 2nd ed., 549 pp., Blackwell, Malden, Mass.

Barr, A. C. (2008), Mobile lid convection beneath Enceladus' south polar terrain, J. Geophys. Res., 113, E07009, doi:10.1029/2008JE003114.

Barr, A. C., and W. B. McKinnon (2007), Convection in Enceladus' ice shell: Conditions for initiation, Geophys. Res. Lett., 34, L09202, doi:10.1029/ 2006GL028799.

Barr, A. C., and L. J. Preuss (2010), On the origin of south polar folds on Enceladus, Icarus, 208, 499-503, doi:10.1016/j.icarus.2010.03.038.

Bassis, J. N., and C. C. Walker (2011), Upper and lower limits on the stability of calving glaciers from the yield strength envelope of ice, Proc. R. Soc. A, 57, 1-17, doi:10.1098/rspa.2011.0422.

Beuthe, M. (2008), Thin elastic shells with variable thickness for lithospheric flexure of one-plate planets, Geophys. J. Int., 172, 817-841, doi:10.1111/j.1365-246X.2007.03671.x.

Bland, M. T., R. A. Beyer, and A. P. Showman (2007), Unstable extension of Enceladus' lithosphere, Icarus, 192, 92-105, doi:10.1016/j.icarus. 2007.06.011.

Bullard, E. C. (1936), Gravity measurements in East Africa, Philos. Trans. R. Soc. London A, 235, 445-531, doi:10.1098/rsta.1936.0008.

Collins, G. C., and J. C. Goodman (2007), Enceladus' south polar sea, Icarus, 189, 72-82, doi:10.1016/j.icarus.2007.01.010.

Colman, S. M., E. B. Karabanov, and C. H. Nelson (2003), Quaternary sedimentation and subsidence history of Lake Baikal, Siberia, based on seismic stratigraphy and coring, J. Sed. Res., 73, 941-956, doi:10.1306/ 041703730941.

Giese, B., R. Wagner, H. Hussmann, G. Neukum, J. Perry, P. Helfenstein, and P. C. Thomas (2008), Enceladus: An estimate of heat flux and lithospheric thickness from flexurally supported topography, Geophys. Res. Lett., 35, L24204, doi:10.1029/2008GL036149.

Gioia, G., P. Chakraborty, S. Marshak, and S. W. Kieffer (2007), Unified model of tectonics and heat transport in a frigid Enceladus, Proc. Natl. Acad. Sci., 1041, 13,578-13,581, doi:10.1073/pnas.0706018104.

Helfenstein, P., et al. (2011), Structural expression of transtension and transpression at the south pole of Enceladus, paper presented at Enceladus Focus Group Meeting, SETI Inst., Mountain View, Calif., 23-24 May.

Howett, C. J. A., J. R. Spencer, J. Pearl, and M. Segura (2011), High heat flow from Enceladus' south polar region measured using $10-600 \mathrm{~cm}^{-1} \mathrm{Cassini}$ CIRS data, J. Geophys. Res., 116, E03003, doi:10.1029/2010JE003718.

Huismans, R. S., Y. Y. Podladchikov, and S. Cloetingh (2001), Dynamic modeling of the transition from passive to active rifting, application to the Pannonian basin, Tectonics, 20, 1021-1039, doi:10.1029/2001TC900010.

Jeffreys, H. (1915), Viscosity of the Earth, Mon. Not. R. Astron. Soc., 75, 648-658.

Kargel, J. S., and S. Pozio (1996), The volcanic and tectonic history of Enceladus, Icarus, 119, 385-404, doi:10.1006/icar.1996.0026.

Lagabrielle, Y., J. Goslin, H. Martin, J.-L. Thirot, and J.-M. Auzende (1997), Multiple active spreading centres in the hot north fiji basin (southwest pacific): a possible model for archaean seafloor dynamics?, Earth Planet. Sci. Lett., 149(1-4), 1-13, doi:10.1016/S0012-821X(97)00060-5.

Mahadevan, L., R. Bendick, and H. Liang (2010), Why subduction zones are curved, Tectonics, 29, TC6002, doi:10.1029/2010TC002720.
Manga, M., and C.-Y. Wang (2007), Pressurized oceans and the eruption of liquid water on Europa and Enceladus, Geophys. Res. Lett., 34, L07202, doi:10.1029/2007GL029297.

McKenzie, D. (1978), Some remarks on the development of sedimentary basins, Earth Planet. Sci. Lett., 40(1), 25-32, doi:10.1016/0012-821X (78)90071-7.

Mitri, G., and A. P. Showman (2008), Thermal convection in ice-I shells of Titan and Enceladus, Icarus, 193, 387-396, doi:10.1016/j.icarus.2007. 07.016 .

Nimmo, F., and R. T. Pappalardo (2006), Diapir-induced reorientation of Saturn's moon Enceladus, Nature, 441, 614-616, doi:10.1038/nature04821. Nimmo, F., and P. Schenk (2006), Normal faulting on Europa: Implications for ice shell properties, J. Struct. Geol., 28, 2194-2203, doi:10.1016/j. jsg.2005.08.009.

Olgin, J. G., B. R. Smith-Konter, and R. T. Pappalardo (2011), Limits of Enceladus's ice shell thickness from tidally driven tiger stripe shear failure, Geophys. Res. Lett., 380, L02201, doi:10.1029/2010GL044950.

Ori, G. G., and V. R. Baker (1995), Geological mechanisms of resurfacing on Venus (Atalanta and Niobe Planitae, Atropos Tessera, Vesta and UT Rupes), Lunar Planet. Sci., XXVI, 1085.

Parkinson, C. D., M-C. Liang, Y. L. Yung, and J. L. Kirschivnk (2008), Habitability of Enceladus: Planetary conditions for life, Origins Life Evol. Biosph., 38, 355-369, doi:10.1007/s11084-008-9135-4.

Patthoff, D. A., and S. A. Kattenhorn (2011), Separating old and young: The south polar dichotomy on Enceladus, Lunar Planet. Sci., XLII, 2700

Porco, C. C., et al. (2006), Cassini observes the active south pole of Enceladus, Science, 311, 1393-1401, doi:10.1126/science.1123013.

Roberts, J. H., and F. Nimmo (2008), Near-surface heating on Enceladus and the south polar thermal anomaly, Lunar Planet. Sci., XXXIX, 1481.

Rudolph, M. L., and M. Manga (2009), Fracture penetration in planetary ice shells, Icarus, 199, 536-541, doi:10.1016/j.icarus.2008.10.010

Sandwell, D., and G. Schubert (2010), A contraction model for the flattening and equatorial ridge of Iapetus, Icarus, 210, 817-822, doi:10.1016/j. icarus.2010.06.025.

Schenk, P. M., and W. B. McKinnon (2009), One-hundred-km-scale basins on Enceladus: Evidence for an active ice shell, Geophys. Res. Lett., 36, L16202, doi:10.1029/2009GL039916.

Schlische, R. W. (1991), Half-graben basin filling models: New constraints on continental extensional basin development, Basin Res., 3(3), 123-141. Schulson, E. M. (1999), The structure and mechanical behavior of ice, J. Miner. Metals Mater. Soc., 51(2), 21-27, doi:10.1007/s11837-999 0206-4.

Sleep, N. H., J. A. Nunn, and L. Chou (1980), Platform basins, Annu. Rev. Earth Planet. Sci., 8, 17-34, doi:10.1146/annurev.ea.08.050180.000313.

Smith-Konter, B., and R. T. Pappalardo (2008), Tidally driven stress accumulation and shear failure of Enceladus's tiger stripes, Icarus, 198, 435-451, doi:10.1016/j.icarus.2008.07.005.

Solomon, S. C., S. K. Stephens, and J. W. Head (1982), On Venus impact basins: Viscous relaxation of topographic relief, J. Geophys. Res., 87, 7763-7771, doi:10.1029/JB087iB09p07763.

Spencer, J. R., A. C. Barr, L. W. Esposito, P. Helfenstein, A. P. Ingersoll, R. Jaumann, C. P. McKay, F. Nimmo, and J. H. Waite (2009), Enceladus: An active cryovolcanic satellite, in Saturn From Cassini-Huygens, edited by M. K. Dougherty, L. W. Esposito, and S. M. Krimigis, pp. 683-724, Springer Netherlands, Heidelberg, Germany, doi:10.1007/978-1-40209217-6-21.

Tanimoto, T. (1997), Bending of spherical lithosphere-Axisymmetric case, Geophys. J. Int., 129, 305-310, doi:10.1111/j.1365-246X.1997. tb01583.x

Tanimoto, T. (1998), State of stress within a bending spherical shell and its implications for subducting lithosphere, Geophys. J. Int., 134, 199-206, doi:10.1046/j.1365-246x.1998.00554.x

Thomas, P. C., et al. (2007), Shapes of the saturnian icy satellites and their significance, Icarus, 190, 573-584, doi:10.1016/j.icarus.2007.03.012.

Timoshenko, S. P., and S. Woinosky-Krieger (1959), Theory of Plates and Shells, 2nd ed., McGraw-Hill, New York.

Tobie, G., et al. (2010), Surface, subsurface and atmosphere exchanges on the aatellites of the outer solar system, Space Sci. Rev., 153, 375-410, doi:10.1007/s11214-010-9641-3.

Turcotte, D. L., and G. Schubert (1982), Geodynamics: Applications of Continuum Physics to Geological Problems, 1st ed., John Wiley, Hoboken, N. J.

Turcotte, D. L., R. J. Willemann, W. F. Haxby, and J. Norberry (1981), Role of membrane stresses in the support of planetary topography, J. Geophys. Res., 86, 3951-3959, doi:10.1029/JB086iB05p03951.

Vening-Meinesz, F. A. (1950), Les grabens africains, resultat de compression ou de tension dans la croute terrestre, Bull. Inst. R. Colon. Belge, $21,539-552$ 
Watts, A. B. (2001), Isostasy and Flexure of the Lithosphere, Cambridge Univ. Press, U. K.

Weissel, J. K., and G. D. Karner (1989), Flexural uplift of rift flanks due to mechanical unloading of the lithosphere during extension, J. Geophys. Res., 94, 13,919-13,950, doi:10.1029/JB094iB10p13919.

Withjack, M. O., R. W. Schlische, and P. E. Olsen (2002), Rift-basin structure and its influence on sedimentary basins, Spec. Publ. SEPM Soc. Sediment. Geol., 73, 57-81, doi:10.2110/pec.02.73.0057.
Yamaoka, K. (1988), Spherical shell tectonics: on the buckling of the lithosphere at subduction zones, Tectonophysics, 147, 179-191, doi:10.1016/ 0040-1951(88)90186-2.

Yamaoka, K. Y. Fukao, and M. Kumazawa (1986), Spherical shell tectonics: Effects of sphericity and inextensibility on the geometry of the descending lithosphere, Rev. Geophys., 24, 27-53, doi:10.1029/ RG024i001p00027. 